\title{
Wider naiven Empirismus und verkleidete Machtansprüche - politische und wissenschaftstheoretische Splitter
}

Viele medizinische Wissenschaftler, auch solche, die sich der Komplementärmedizin widmen, gehen von etwa folgendem Welt- und Wissenschaftsbild aus: Es gibt eine Wirklichkeit, die sich irgendwie «draussen» befindet. Durch unsere Sinnesorgane haben wir mehr oder weniger zuverlässige Kenntnis über diese Wirklichkeit. Was die Wissenschaft von der Alltagswelterfahrung unterscheidet, ist die Tatsache, dass sie ihre Möglichkeiten der Erfahrung dieser Aussenwelt stärker absichert. Zum einen durch kollektiven Konsens, zum anderen durch Erkenntnismethoden. Diese Methoden sind ein bisschen wie kollektive Sinnesorgane. Was einmal festgestellt ist, das ist so. Sind die Fidschi-Inseln einmal entdeckt und kartographiert, dann bleiben sie auf der Landkarte. Weiss man einmal, an welchen Positionen sich die Planeten befinden und welchen Gesetzmässigkeiten ihre Umlaufbahnen gehorchen, dann kann man ihre Bewegungen berechnen und ihre zukünftigen Positionen voraussagen. Tatsache ist, wie Wittgenstein erkannte, alles, was der Fall ist, und das kann man fest-stellen. Dann weiss man es. $\mathrm{Zu}$ dieser Fest-stellung verwendet die Wissenschaft ihre Methoden. Und was einmal festgestellt ist, ist der Fall und gehört zu unserem Wissen. Es ist ein einfaches Folgegesetz, dass sich unser Wissen somit laufend erhöht, mindestens potenziell, auch wenn nicht jede einzelne Person über alles Wissen verfügt, so doch die Wissenschaftlergemeinschaft oder die Menschheit als Ganzes. Wissen ist also überdies kumulativ.

Deshalb wissen wir, dass z.B. bestimmte Arzneimittel wirksam sind, nämlich diejenigen, für die der Wirksamkeitsnachweis erbracht wird, bei anderen Methoden wissen wir es nicht genau, nämlich weil sie nicht gut genug untersucht sind, und bei wieder anderen wissen wir, dass sie unwirksam sind, weil sie nämlich ihre Wirksamkeit nicht unter eindeutigen Beweis stellen konnten. Deshalb kann man auch bestimmte Heilmethoden und Arzneimittel unter Berufung auf mangelnde wissenschaftliche Belege aus der öffentlichen Erstattungsfähigkeit ausgrenzen, andere nicht. Alles unter Berufung auf Wissenschaftlichkeit, denn was könnten sonst die Kriterien sein?
Deswegen wurde etwa vom Bundesausschuss für Ärzte und Krankenkasse der Akupunktur der Beweis ihrer Wirksamkeit abgesprochen, die sie nun in den grossen Erprobungsverfahren in randomisierten, Plazebo-kontrollierten Studien unter Beweis stellen muss. Deswegen werden jetzt die meisten naturheilkundlichen Medikamente bis auf wenige Ausnahmen von der Positivliste gestrichen. Deswegen halten die meisten Vertreter der akademischen Medizin und ärztliche Standesvertreter viele Verfahren der Komplementärmedizin für unwirksam.

Sie unterstellen bei solchen Bewertungen in den meisten Fällen ein Wissenschafts- und Weltbild, das ich oben kurz skizziert habe und das ich «naiv-empiristisch» nennen will. «Naiv» deswegen, weil es in den seltensten Fällen wirklich begründet und reflektiert ist, sondern sich an einen vermeintlichen Allgemeinkonsens anlehnt, und «empiristisch» deswegen, weil es von einer stabilen, unabhängig von menschlichen Erkenntnisbestrebungen vorhandenen und gleichbleibenden Aussenwelt ausgeht.

Das Verständnis von Wissenschaft, das einer solchen Argumentation zugrunde liegt, ist sachlich kaum belegbar, wird innerhalb der wissenschaftstheoretischen Diskussion schon längst nicht mehr ernsthaft diskutiert und ist trotzdem noch immer sehr wirkmächtig. Woran liegt das? Und wie würde ein erweitertes Verständnis aussehen?

Bleiben wir zuerst bei der ersten Frage, bei den Gründen für das Beharren auf einem sachlich falschen und hinderlichen Verständnis von Wissenschaft. Dafür gibt es mehrere Gründe. (1) Einer ist sicherlich der, dass der Prozess der Reflexion über Wissenschaft und wissenschaftliche Methoden und Voraussetzungen, als Wissenschaftstheorie oder Wissenschaftsforschung eine junge Disziplin ist. Kaum ein aktiver Forscher ist es gewöhnt, Erkenntnisse über den Forschungsprozess zur Kenntnis zu nehmen, geschweige denn selbst darüber nachzudenken, welche Voraussetzungen seiner täglichen Arbeit zugrunde liegen und ob diese begründet sind. Unsere Ausbildungs- und Lehrstrukturen sehen diesen Reflexionsprozess

\begin{tabular}{|c|c|c|}
\hline KARGER & (C) 2004 S. Karger GmbH, Freiburg & $\begin{array}{l}\text { PD Dr. Dr. phil. Harald Walach } \\
\text { Klinikum der Universität }\end{array}$ \\
\hline $\begin{array}{l}\text { Fax +49 } 7614520714 \\
\text { E-mail Information@Karger.de } \\
\text { www.karger.com }\end{array}$ & $\begin{array}{l}\text { Accessible online at: } \\
\text { www.karger.com/fkm }\end{array}$ & $\begin{array}{l}\text { Institut für Umweltmedizin und Krankenhaushygiene } \\
\text { Hugstetter Str. 55 } \\
\text { D-79106 Freiburg } \\
\text { Tel. +49 } 761 \text { 270-5497/98, Fax -7224 } \\
\text { E-mail walach@ukl.uni-freiburg.de }\end{array}$ \\
\hline
\end{tabular}


nicht vor und delegieren ihn an Fachdisziplinen, wie eben die Philosophie oder die Wissenschaftsforschung. Ein anderer Grund mag darin liegen, dass die Wissenschaft als kollektiver Gesamtprozess konservativ und träge ist, und dies ist auch ganz gut so. Denn wäre sie das nicht, so müssten wir uns fortlaufend der Gefahr aussetzen, potentiell falsche oder gar gefährliche Verfahrensweisen als wissenschaftlich akzeptierte über uns ergehen zu lassen. Bereits jetzt wird für mein Gefühl einem neuen Verfahren viel zu schnell der Stempel der wissenschaftlichen Dignität aufgedrückt. Einige Skandale der jüngeren Vergangenheit belegen dies, wie etwa die vorschnelle und unreflektierte Gabe von Hormonen zur Osteoporoseprävention ohne Rücksicht auf mögliche schädliche Wirkungen, oder der Contergan-Skandal. Wissenschaft muss aus genau diesen Gründen eine Komponente der Skepsis, der Kontrolle, der Gemächlichkeit, ja der Zögerlichkeit haben, um genau solche Fehler nach Möglichkeit zu vermeiden. Das allein darf aber nicht als Vorwand oder gar Begründung für die mangelnde Zurkenntnisnahme wichtiger grundlegender Erkenntnisse verwendet werden.

(2) Ein systematischerer Grund dürfte darin zu suchen sein, dass unsere gesamte akademische Ausbildung radial organisiert ist, mit relativ wenig Querverbindungen: man studiert ein Fach und arbeitet sich innerhalb dieses Faches immer mehr in die Tiefe. Für befruchtende Querverbindungen bleibt immer weniger Platz und Zeit, und je weiter man in die Tiefe dringt, desto spezialisierter wird das Wissen und desto weniger Berührung zu anderen Disziplinen und Erkenntnissen hat man. Bildlich ausgedrückt: Wäre unser Ausbildungssystem ein Spinnennetz, dann wären Querverbindungen zwischen den radial nach aussen laufenden Fäden noch ganz innen, also etwa auf dem Niveau der Schule und der einleitenden Kurse gegeben, aber schon hier nicht mehr ganz durchgehend. Je weiter sich der radiale Strang aber vom Zentrum entfernt, desto weniger durchgehend, seltener und brüchiger werden die Querverbindungen. Eine Spinne, die ihr Netz tatsächlich so weben würde, wie unser Ausbildungssystem beschaffen ist, hätte wenig Chancen, ausreichend Beute zu machen oder müsste ein vorzeitiges Reissen ihres Netzes in Kauf nehmen. Nun ist aber unser Wissenschaftssystem genau so aufgebaut, dass Fachspezialisten zwar in ihrem Fach Koryphäen, in Nebendisziplinen oder gar in grundlegenden Fragen Analphabeten sind. Hier kommt uns eine dumme menschliche Angewohnheit in die Quere: Wir generalisieren gern. Wenn es uns an einem Ort gefallen hat, dann meinen wir, andere Orte in der Nähe sind auch schön. Wenn wir einmal ein Gericht gegessen haben, das uns den Magen verdorben hat, werden wir es auch zu anderen Zeiten und Orten meiden. Wenn wir Menschen aus anderen Ländern kennen gelernt haben, die uns missfallen haben, werden wir tendenziell diese Erfahrung auf andere Menschen aus diesem Land übertragen. Wenn sich ein Wissenschaftler auf einem Gebiet einen Namen gemacht hat, meinen wir, er hat auch auf anderen etwas zu sagen. Das Gegenteil ist häufig der Fall, denn die vertiefte Kenntnis in einem Gebiet verträgt sich in den sel- tensten Fällen mit einer breiten Kenntnis und umgekehrt. Unser Ausbildungssystem fördert also radial vertieftes Wissen, auf Kosten einer axialen Verankerung, wie das eben die Spinne tut, um ihr Netz zu stabilisieren.

(3) Ein anderer Grund, weswegen die neueren Erkenntnisse der Wissenschaftsforschung nicht aufgegriffen werden, die jenem naiven Empirismus gefährlich werden würden, der fast überall in der medizinischen Forschung herrscht, dürfte ein systematischer sein. Die herrschende Epistemologie ist auch bestimmten Interessen dienlich. Kritiker dieses empiristischen Modells, angefangen von Husserl und Heidegger und deren Nachfolger im Konstruktivismus französischer Schule bis hin zu Vertretern der kritischen Theorie der Frankfurter Schule haben darauf hingewiesen, dass die Vorherrschaft dieses empiristischen Modells interessengestützt und -geschützt ist. Dieses Welt- und Wissenschaftsmodell verschleiert nämlich ganz famos, wie stark unsere Erkenntnisweise der Welt von bestimmten Vorbedingungen abhängig ist, die alles andere als selbstverständlich sind. Wenn wir etwa unseren Körper als eine komplexe Maschine sehen, die im Wesentlichen kausalmechanistisch funktioniert, dann werden wir alles ausblenden, was nicht in dieses Bild passt, und die Wirklichkeit so partitionieren und präparieren - um mir eine Vokabel aus dem physikalisch-epistemologischen Wortschatz auszuleihen, die dieses aktive Eingreifen festhält -, dass wir sie als Mechanismus sehen. Wir können dies am klassischen Beispiel einer Infektion deutlich machen. Es ist klarerweise so, dass bei Infektionen Erreger im Spiel sind, Viren oder Bakterien, die mehr oder weniger aggressiv sind. Es gibt solche, die sind so aggressiv, dass sie sehr kausal eine Erkrankung verursachen, und zwar beinahe bei jedermann, obwohl es auch bei diesen ganz aggressiven Erregern Ausnahmen geben wird. Es gibt aber auch solche Erreger, die manchmal eine Infektion hervorrufen, manchmal nicht, bei manchen Menschen zu Erkrankungen führen, bei anderen nicht. Sollen wir sie im einen Fall als Ursache der Erkrankung behandeln, im anderen Fall nicht? Warum fokussieren wir unsere Anstrengung der Erkenntnis und Behandlung einzig und allein auf den Erreger als Ursache? Genauso gut könnten wir die Wirklichkeit anders aufteilen und ordnen. Wir könnten z.B. unseren Organismus weniger als Maschine betrachten, sondern als ein komplexes, sich selbst organisierendes System, wie das T. Esch in dieser Zeitschrift im letzten Beitrag zum Schwerpunktthema Gesundheit 2003 getan hat [1], oder ich selbst im Rückgriff auf Konzepte von Uexkülls [2]. Dann rückt nicht das vermeintliche Pathogen in den Blickpunkt, sondern der Wirtsorganismus: Warum wird er gerade jetzt für den Erreger anfällig? Warum für diesen, und nicht für einen anderen? Wie lässt sich die Abwehrkompetenz des Organismus steigern? Dies sind andere, komplexere Fragen als die nach der Natur des Erregers und wie er $\mathrm{zu}$ hemmen ist. Vor allem werden eine andere Handlungskompetenz verlangt und andere Interventionen nahe gelegt. Dann gibt man nicht einfach ein Antibiotikum, sondern versucht den Organismus durch komplexe Verfahren zu Eigen- 
leistungen zu stimulieren. Das kann ein klassisch naturheilkundlicher oder balneologischer Ansatz sein, eine Homöopathie- oder Akupunkturbehandlung, oder eine komplexe Intervention, die Fragen der Lebensführung, der Diät und der Expositionsvermeidung beinhaltet. In jedem Fall aber wird die Handlungskompetenz an den Patienten zurückgegeben und dieser für die weitere Genesung oder Prävention in die Verantwortung gezogen.

Das mechanistische Modell des Körpers, in dem nach dem Übeltäter gefahndet wird, der eine Krankheit verursacht hat, dient weniger den Patienten, als dem medizinisch-pharmazeutischen Komplex, wie ich die Allianz aus moderner Pharmaforschung und akademischer Medizin nennen möchte. Denn wenn es so etwas wie die Fiktion einer simplen Krankheitsverursachung «wirklich» gibt, einen Keim, ein Gen, das aus dem Ruder läuft, ein Entzündungsprozess, der überbordet, dann gibt es auch «wirklich» eine einfache Therapie: den Erreger töten, das Gen zurückregulieren, den Entzündungsprozess stoppen, usw. Und wie tut man dies am einfachsten? Mit Arzneimitteln selbstverständlich, und zwar am besten und liebsten mit den ganz modernen, massgeschneiderten, die eben die Feinheiten der differenzierten Rezeptorgarderobe in ihr kausalistisches Verständnis integriert haben und eben nicht das gesamte Immunsystem herunterregulieren, sondern eben nur ein bestimmtes Interleukin mit einem gezielten monoklonalen Antikörper. Allen Verfeinerungen des kausalistischen, empiristischen Weltmodells zum Trotz bleibt eine Konsequenz bestehen: die Suche nach Ursachen für Krankheiten wird fortgesetzt und ihre Bekämpfung mit Mitteln der modernen Pharmakologie auch. Der Tatsache zum Trotz, dass unser Gesundheitssystem pleite und in Zukunft unbezahlbar ist. Der Tatsache zum Trotz, dass genau dieses Weltmodell sachlich eine Abstraktion und Konstruktion ist, die man mit gutem Grunde genau anders durchführen kann. Warum? Eben weil die Beibehaltung dieses Modells Interessen dient. Es dient dem pharmazeutischen Komplex, weil es immer weiter zur Erfindung neuer Medikamente anregt, die immer mehr die Schlüssel für die vermeintlich immer genauer erkannten Schlösser sein sollen. Dadurch, dass die Entwicklungskosten immer höher werden und verstärktes Sicherheitsbedürfnis immer komplexere Regulationsprozeduren fordert, können solche Medikamente nur noch entwickelt werden, wenn sie auch genügend Geld einspielen, also - zuhanden der Öffentlichkeit - gewinnbringend verkauft werden können. Die Beibehaltung dieses Modells dient aber auch dem ärztlichen Stand als ganzem.

Erlauben wir uns für einige Momente die Visualisierung eines Idealtraums: Menschen werden auch in unserer idealen Welt krank werden. Aber sie sind sich in diesem Falle bewusst, dass die Erkrankung ein komplexer Prozess ist, zu dem sie auch selbst einen kleinen Teil beigesteuert haben. Sie wissen zwar, dass die Bekämpfung eines Erregers «kausal» möglich, oft nötig, oft aber auch kurzsichtig ist. Sie wissen darum, dass sie durch ihr Verhalten, ihre Art des Umgangs mit sich und mit anderen zu Gesundheit und Krankheit mit beitragen. Ärzte wissen, dass im Akutfall eine Erregerbekämpfung nötig sein kann. Sie wissen aber auch, dass die beste Therapie die Stärkung der Kompetenz des Patienten ist: des Immunsystems durch entsprechende Verfahren, die Prävention chronischer Krankheiten durch sparsamen Umgang mit invasiven Interventionen und nebenwirkungsbehafteten Medikamenten. Weil ihre Verfahren erfolgreich sind, haben ihre Patienten ihre Dienste tendenziell immer weniger nötig. Im idealen Land der Gesundheit ist für einen Arzt als gut verdienenden Überbeschäftigten gar kein Platz, weil er nämlich seine Patienten in der Regel gesund erhält und diese dann irgendwann eines hoffentlich geruhsamen Todes sterben, dessen Feststellung dann die letzte Amtshandlung eines guten Arztes ist, der sich selbst abgeschafft hat.

Allein die Tatsache, dass wir uns ein solches Szenario kaum vorstellen können, geschweige denn daran arbeiten, dass es Wirklichkeit wird, zeigt, wie sehr uns das herrschende Modell im Griff hat. Es war nicht immer so. Chinesische Ärzte und auch griechische wurden früher dafür bezahlt, dass sie ihre $\mathrm{Pa}$ tienten gesund erhielten und hatten deswegen ein Interesse daran, dass sie eben nicht krank wurden. Denn in diesem Fall wurden sie nicht mehr bezahlt und mussten zusehen, dass die Patienten schnell wieder gesund wurden. Stellen wir uns ein solches Bild bei uns vor: Es gibt eine gewisse Anzahl Ärzte, die aufgrund der Bevölkerungsdichte festgelegt wird. Diese erhalten ein Gehalt aus der öffentlichen Kasse dafür, dass sie die Patienten in ihrem Umkreis gesund erhalten. Jeder Kranke schmälert ihr Einkommen. Medikamente, die sie verabreichen, bezahlen die Ärzte selbst (nur als Zwischenbemerkung: der homöopathische Zauberkünstler, der seine Patienten mit einer gut gezielten Hochpotenz in Einmaldosis bei Laune hält, hätte damit finanziell gesehen das grosse Los gezogen), usw. Man sieht sehr leicht ein, dass ein solches Konzept wenig Raum liesse für die Bereicherungsmaschine auf öffentliche Kosten, die der pharmazeutisch-medizinische Komplex zur Zeit darstellt. Es liesse allerdings sehr viel Spielraum für komplexe, stimulierende Verfahren, die die Kompetenz, die Abwehrfähigkeit und die allgemeine Robustheit des Organismus stärken, wie dies viele Verfahren der Naturheilkunde und der Komplementärmedizin tun. Plötzlich würden ganz andere Fragen gestellt. Weniger die Frage: «Wirkt Akupunktur als Plazebo?», sondern vielleicht: «Ist Akupunktur langfristig gesehen geeignet, die Lebensqualität zu verbessern, Arbeitsausfälle zu reduzieren, Patientenzufriedenheit zu erhöhen?» Die Daten unserer Evaluation der Erprobungsverfahren der Innungskrankenkasse weisen darauf hin, dass dies tatsächlich der Fall ist [3]. Weniger die Frage: «Welches Medikament senkt hohen Blutdruck möglichst schonend?» würde dann im Zentrum des Interesses stehen, sondern mehr die Frage: «Wie kann man verhindern, dass Menschen überhaupt hohen Blutdruck entwickeln?» Wäre es vielleicht hilfreich, wenn sie ein Element der Besinnung und Meditation in ihren täglichen Lebensablauf integrieren? In einem solchen System würde weniger Krankheit und Kranksein belohnt, wie in unserem heuti- 
gen - Gesunde bezahlen, Kranke erhalten Leistung und $\mathrm{Zu}$ wendung -, sondern Gesundsein würde belohnt und Kranksein würde systemisch-psychologisch «bestraft» werden. Ich überlasse es an dieser Stelle den Lesern, über Konsequenzen des herrschenden Welt- und Wissenschaftsbildes nachzudenken und sich Alternativen auszudenken und Visionen zu entfalten. Wenn wir Glück haben, ist vielleicht auch einmal ein verantwortlicher Gesundheitspolitiker unter den Lesern.

Ich wende mich stattdessen zum Abschluss der versprochenen Frage zu, wie denn ein alternatives, sachgerechteres Bild von Wissenschaftsprozess und Welt zu gewinnen ist und welche Konsequenzen sich daraus ergeben. Ein bisschen habe ich schon vorweggenommen: Ein angemesseneres Verständnis von Wissenschaft und Welt anerkennt, dass unsere Wahrnehmung immer gestaltend mit der Wirklichkeit in Wechselwirkung tritt. Es wäre töricht, zu verneinen, dass es so etwas wie eine Wirklichkeit gibt. Aber es ist gleichzeitig extrem naiv zu glauben, die Wirklichkeit sitze vor unserer Nase wie ein Frosch, den man entweder rechtzeitig fängt oder der einem entwischt. Wir interagieren mit unserer Umwelt und gestalten sie damit. Unser Erkenntnisprozess selber, die Methoden und Mittel die wir anwenden, definieren, was wir erkennen. Watzlawik hat das einmal mit dem Spruch illustriert, die Welt würde für den, der nur mit einem Hammer ausgerüstet ist, nur aus Nägeln bestehen. Wenn wir definieren, dass nur die Erkenntnis eines pathogenen Erregers und seine Bekämpfung von Interesse ist, dann werden wir genau diese Erkenntnis gewinnen, oder auch nicht. Wenn wir aber beginnen andere Fragen zu stellen, erhalten wir auch andere Antworten. Durch unsere Art und Weise der Aufteilung der Welt in interessant und uninteressant, in wichtig und unwichtig, relevant und irrelevant, prägen wir vor, was wir erkennen können und was nicht. Ein moderneres, angemesseneres Konzept von Erkenntnis und Wissenschaft besagt, dass Wissenschaft ein komplexer sozialer Prozess ist, in dem das, was wichtig ist, laufend neu verhandelt wird. Prozesse sozialer Einflussnahme und Geltung, das Spiel wirtschaftlicher und politischer Interessen, definieren «was der Fall ist», was wichtig ist und was beforscht wird. Einzelne haben in diesem sozialen Gefüge wenig Chancen, etwas zu verändern. Aber Randgruppen, die sich formieren und auf ein vernachlässigtes Problem oder eine Anomalie hinweisen, haben eine Chance. Vor allem dann, wenn sich der sog. Mainstream verrannt hat und in wichtigen Problemen nicht mehr weiterkommt. Dann ist die Zeit der Aussenseiter gekommen, die, wenn sie wirkliche Lösungen für wichtige Probleme anzubieten haben, auch irgendwann gehört werden. Sozial orientierte Wissenschaftstheoretiker seit Kuhn haben immer wieder darauf hingewiesen, dass gerade Anomalien und Randbereiche für den Wissenschaftsprozess dann wichtig werden, wenn sie aus Sackgassen herausführen. Während Thomas S. Kuhn mehr oder weniger irrationale und unvorhersehbare Prozesse für diese Veränderungen verantwortlich machte $[4,5]$, rücken moderatere und auch pragmatischere Nachfolger oder Kritiker seines Denkens gelungene Problem- lösungen [6] oder soziale Gruppenbildungsprozesse [7] ins Interesse der Aufmerksamkeit. Immer noch gilt das Wort Ludwik Flecks: «Eine wissenschaftliche Tatsache ist die Übereinkunft, mit dem Denken aufzuhören» [8]. Die Wirklichkeit und was wir für sie halten ist nicht gegeben, sondern wird von uns gestaltet in einem komplexen sozialen Prozess, den wir Wissenschaft nennen.

Das ist zugleich die Chance der Komplementärmedizin in der heutigen Gesundheitskrise. Der Hauptstrom der medizinischen Forschung stösst in der Endlichkeit unserer Ressourcen mit dem momentan gültigen Paradigma an Grenzen, und Alternativen sind gerade jetzt gefragt. Die Komplementärmedizin hat, als gesamte Bewegung, Lösungen für Probleme anzubieten, die von der herrschenden Auffassung nur schwer zu beherrschen sind. Das bietet die Möglichkeit der Integration. Aber eines ist dafür Voraussetzung: Dass die Komplementärmedizin ihren eigenen paradigmatischen Wurzeln treu bleibt und nicht einfach und unkritisch das Welt- und Wissenschaftsmodell übernimmt, das genau jene Probleme erzeugt, die komplementärmedizinische Ansätze lösen könnten. Das ist kein Plädoyer für verschnupftes Fernestehen. Aber es ist ein Plädoyer dafür, nicht unkritisch das Lied derer zu singen die meinen, sie würden den Takt angeben. Ich kann es mir an dieser Stelle nicht verkneifen, Brecht zu paraphrasieren. Er hat in einer seiner Geschichten von Herrn K. die Geschichte vom Agenten erzählt: Zu Herrn K. kam eines Tages ein Agent einer fremden Macht mit einer Vollmacht, in der stand, jeder solle ihm zu Diensten sein. Der Agent fragte Herrn K.: «Willst Du mir dienen?». Herr K. bat ihn in sein Haus, gab ihm zu essen, gab ihm eine Decke und einen Platz zu schlafen. Und als der Agent nach Jahren von der guten Pflege alt und fett geworden war, starb er. Herr K. wickelte ihn in seine Decke, warf ihn vor die Tür und sagte: «Nein».

H. Walach, Freiburg i. Br.

\section{Literatur}

1 Esch T: Stress, Anpassung und Selbstorganisation: Gleichgewichtsprozesse sichern Gesundheit und Überleben. Forsch Komplementärmed Klass Naturheilkd 2003;10:330-341.

2 Walach H: Ganzheitliche Heilkunde - theoretische Überlegungen und der Versuch einer Vision; in Albrecht H (Hrsg): Heilkunde versus Medizin? Gesundheit und Krankheit aus der Sicht der Wissenschaften. Stuttgart, Hippokrates, 1993, pp 117-147.

3 Güthlin C, Lange O, Walach $\mathrm{H}$ : Measuring the effects of acupuncture and homoeopathy in general practice: An uncontrolled prospective documentation approach. BMC Public Health 2004;4.

4 Kuhn TS: Die Entstehung des Neuen - Studien zur Struktur der Wissenschaftsgeschichte. Frankfurt, Suhrkamp, 1977.

5 Kuhn TS: Die Struktur wissenschaftlicher Revolutionen. Frankfurt, Suhrkamp, 1967.

6 Laudan L: Progress and Its Problems: Towards a Theory of Scientific Growth Berkeley, University of California Press, 1977.

7 Collins H, Pinch T: The Golem. What Everyone Should Know about Science. Cambridge, Cambridge University Press, 1993.

8 Fleck L: Entstehung und Entwicklung einer wissenschaftlichen Tatsache. Einführung in die Lehre vom Denkstil und Denkkollektiv. Mit einer Einleitung herausg. von L. Schäfer und T. Schnelle. Frankfurt, Suhrkamp, 1980 (Original erschienen 1935). 